Symmetry Modes Summary for Cmce to Pbca phase transition.

\begin{tabular}{|l|l|l|}
\hline Atoms & WP & \multicolumn{1}{|c|}{ Modes } \\
\hline $\mathrm{Cl} 3$ & $16 g$ & $\mathrm{GM} 1+(3) \mathrm{Y} 2+(3)$ \\
\hline $\mathrm{Cl} 1 \mathrm{Cl} 4 \mathrm{Cd} 1$ & $8 f$ & $\mathrm{GM} 1+(2) \mathrm{Y} 2+(\mathbf{1})$ \\
\hline $\mathrm{Cl} 2$ & $8 e$ & $\mathrm{GM} 1+(1) \mathrm{Y} 2+(2)$ \\
\hline
\end{tabular}

Note: The primary mode is written in bold letters

\title{
Summary of Amplitudes
}

\begin{tabular}{|l|l|l|l|c|c|}
\hline K-vector & Irrep & Direction & $\begin{array}{l}\text { Isotropy } \\
\text { Subgroup }\end{array}$ & Dimension & Amplitude (̊) \\
\hline$(0,0,0)$ & GM1+ & (a) & Cmce (64) & 10 & $0.237(2)$ \\
\hline$(1,0,0)$ & Y2+ & (a) & Pbca (61) & 8 & $1.565(2)$ \\
\hline
\end{tabular}

Global distortion: $1.5833 \AA$

Symmetry Modes Summary for $P b c a$ to $P 2_{1} 2_{1} 2_{1}$ phase transition

\begin{tabular}{|l|l|l|}
\hline Atoms & WP & \multicolumn{1}{|c|}{ Modes } \\
\hline Cl1 Cl5 Cd1 Cl3 Cl4 Cl2 & $8 c$ & GM1+(3) GM1-(3) \\
\hline Cd2 & $4 a$ & GM1-(3) \\
\hline
\end{tabular}

Note: The primary mode is written in bold letters

\section{Summary of Amplitudes}

\begin{tabular}{|l|l|l|l|c|c|}
\hline K-vector & Irrep & Direction & $\begin{array}{l}\text { Isotropy } \\
\text { Subgroup }\end{array}$ & Dimension & Amplitude ( \\
\hline$(0,0,0)$ & GM1+ & (a) & Pbca (61) & 18 & $0.183(3)$ \\
\hline$(0,0,0)$ & GM1- & (a) & P2_12_12_1(19) & 21 & $1.008(3)$ \\
\hline
\end{tabular}

Global distortion: $1.0244 \AA$ 